\title{
SOME EXAMPLES IN SHAPE THEORY USING THE THEORY OF COMPACT CONNECTED ABELIAN TOPOLOGICAL GROUPS
}

\author{
BY \\ JAMES KEESLING $\left({ }^{1}\right)$
}

\begin{abstract}
In previous papers the author has studied the shape of compact connected abelian topological groups. This study has led to a number of theorems and examples in shape theory. In this paper a theorem is proved concerning the Čech homology of compact connected abelian topological groups. This theorem together with the author's previous results are then used to study the movability of general compact Hausdorff spaces. In the theory of shape for compact metric spaces, a number of significant theorems have been proved for movable compact metric spaces. Among these are a theorem of Hurewicz type due to K. Kuperberg, a Whitehead type theorem due to Moszyńska, and a theorem concerning the exactness of the Čech homology sequence for movable compact metric pairs due to Overton. In this paper examples are constructed which show that these theorems do not generalize to arbitrary movable compact Hausdorff spaces without additional assumptions.
\end{abstract}

Introduction. Let $C$ denote the category of compact Hausdorff spaces and continuous maps and let $H: C \rightarrow H C$ be the homotopy functor. Let $S: C \rightarrow S C$ denote the shape functor to the shape category $S C$. This notation is basically that of W. Holsztyniski [7] and that of S. Mardesić [21]. We will also be studying the shape of pointed compact Hausdorff spaces and compact Hausdorff pairs. In these cases we will let $C$ denote the category of pointed compact Hausdorff spaces (respectively, compact Hausdorff pairs), $H$ the respective homotopy functor, and $S: C \rightarrow S C$ the respective shape functor to the respective shape category. We will let context make clear which category and which shape theory is under consideration. We should caution the reader, however, that whether a space has a certain shape property or not may depend on the category under consideration. A continuum $X$ may be movable, but this does not allow us to say that for some choice of $x \in X$, the pointed continuum $(X, x)$ is movable. Of course, if $(X, x)$ is movable, then so is $X$.

Received by the editors January 6, 1975.

AMS (MOS) subject classifications (1970). Primary 55D99, 55B05.

Key words and phrases. Shape theory, movable space, Čech homology, Hurewicz type theorem, Whitehead type theorem, exactness of Čech homology, compact connected abelian topological group.

(1)Supported by NSF grant GP-42664. 
We will only be concerned about shape theory for compact Hausdorff spaces and so will not need the more general theory for arbitrary topological spaces by Mardesic in [21]. One of the most useful properties of the shape functor for compact Hausdorff spaces is that it is continuous ([7] and [21]). This property was used by Holsztyniski to give an axiomatic characterization of the shape functor [7]. This property was used by the author in his study of the shape of compact connected abelian topological groups ([8], [9], [10], and [11]). In [12] the continuity of $S: C \rightarrow S C$ was used to show that $S$ preserves direct products. In [12] an example was given which shows that the more general shape functor defined on all topological spaces introduced by Mardesić [21] does not preserve direct products. The continuity of the shape functor and the continuity of Čech homology and Čech cohomology for the category of compact Hausdorff spaces will be used a number of places in this paper.

The shape invariant notion of movability was introduced by $\mathbf{K}$. Borsuk in [2]. Borsuk introduced the notion for the class of compact metric spaces. Using the ANR-systems approach to shape theory Mardesic and Segal [24] generalized the notion of movability to the category of compact Hausdorff spaces and by analogy to the category of pointed compact Hausdorff spaces and compact Hausdorff pairs. Other generalizations of movability to a larger class of spaces than the compact metric spaces were introduced by M. Moszyńska [26] and Kozlowski and Segal [17]. The different notions of movability agree on the class of compact metric spaces (see [17] and [32]). However, these notions of movability do not agree in the category of compact Hausdorff spaces.

The notion of movability has played an important role in the development of shape theory. The class of movable spaces is significantly larger than the class of ANR's and yet a number of theorems concerning ANR's generalize in shape theory to the class of movable spaces. Among these is a shape version of the Whitehead theorem ([27] and [13]), a theorem of Hurewicz type [19], and a theorem concerning the exactness of Cech homology for movable compact metric pairs [28]. These theorems will be stated precisely in $\$ 1$ of the paper. In each of these theorems, the spaces involved were not only assumed to be compact, but were also assumed to be metric. In this paper we use the author's results in his study of the shape of compact connected abelian topological groups $([8],[9],[10]$, and [11]) to show that the assumption of metrizability in each of these cases cannot be eliminated without additional assumptions. We also give some compact metric examples which help to illustrate and show the limits of these theorems in the compact metric case as well. The main examples described in $\S \S 3,4$, and 5 were announced with a brief description in [14].

The reader is assumed to have a basic knowledge of shape theory, particularly of the approach described by Mardesic in [21] and the ANR-systems 
approach due to Mardesic and Segal [25]. The reader should also be familiar with the basic elements of the theory of compact topological groups. In particular he should be familiar with Pontryagin duality theory for locally compact abelian topological groups. Pontryagin [30] is a good reference. Use also will be made in this paper of the theory of abelian groups. The volumes by Fuchs will contain all the material we need in this area ([4] and [5]).

If $(X, x)$ is a pointed compactum, we let $\pi_{k}(X, x)$ denote the $k$-dimensional shape group of $(X, x)$. We let $\pi_{k}\{(X, x)\}$ denote the $k$-dimensional homotopy pro-group of $(X, x)$. This notation is the same as in [13] and [3]. Both $\underline{\pi}_{k}(X, x)$ and $\pi_{k}\{(X, x)\}$ are shape invariants of $(X, x)$ where one uses pointed shape theory. In $\S 4$ we point out that for $y \neq x, \pi_{k}(X, x)$ and $\underline{\pi}_{k}(X, y)$ and $\pi_{k}\{(X, x)\}$ and $\pi_{k}\{(X, y)\}$ may not be isomorphic, respectively, even for $X$ a metric continuum.

We let $H_{k}(X)$ and $H^{k}(X)$ denote the $k$-dimensional Čech homology and cohomology groups of $X$ with integer coefficients. These are shape invariants of $X$ whether $X$ is a compact Hausdorff space, a pointed compact Hausdorff space, or a compact Hausdorff pair.

1. Movability. In [2] Borsuk introduced the notion of movability for compact metric spaces. In [25] Mardesic and Segal translated this concept into the language of ANR-systems and generalized the concept to arbitrary compact Hausdorff spaces. We now give the definition of movability due to Mardesić and Segal.

1.1. Definition. Let $X$ be a compact Hausdorff space and $\underline{X}=\left\{X_{\alpha}\right.$; $\left.\pi_{\alpha \beta} ; \alpha \leqslant \beta \in A\right\}$ be an associated ANR-system for $X$. Then $X$ is said to be movable provided that for $\alpha \in A$, there is a $\beta \geqslant \alpha$, such that for every $\gamma \in A$ with $\gamma \geqslant \beta$, there is a map $r^{\beta \gamma}: X_{\beta} \rightarrow X_{\gamma}$ such that

$$
H\left(\pi_{\alpha \gamma} \circ \gamma^{\beta \gamma}\right)=H\left(\pi_{\alpha \beta}\right)
$$

where $H$ is the homotopy functor. If $X$ has a movable ANR-system associated with it, then $X$ is said to be movable. In [24] Mardesic and Segal show that the definition of movability of a compactum $X$ is independent of the ANR-system associated with it and is a shape invariant of $X$. This definition and result is also valid for pointed compact Hausdorff spaces and for compact Hausdorff pairs.

All ANR's are movable as are all plane compacta and all 0-dimensional compacta. If one takes an arbitrary collection of movable compacta, then the product space is also movable. There are nonmovable peano continua (see [10] for one construction). However, every one-dimensional peano continuum is movable [29].

A compact connected abelian topological group is movable if and only if it is locally connected [8]. Moreover, this is not true for compact connected topological groups which are not abelian. They may be locally connected and not movable. 
We now state several important theorems concerning movable spaces. The first is a shape version of the Whitehead theorem due to Moszyniska [27].

1.2. TheOREM. Let $(X, x)$ and $(Y, y)$ be finite-dimensional movable pointed continua and let $F:(X, x) \rightarrow(Y, y)$ be a shape morphism such that $F_{*}: \pi_{k}(X, x)$ $\rightarrow \underline{\pi}_{k}(Y, y)$ is an isomorphism for $1 \leqslant k<n_{0}+1=\max \{1+\operatorname{dim} X, \operatorname{dim} Y\}+1$ and an epimorphism for $k=n_{0}+1$. Then $F$ is a shape equivalence.

There is a gap in the proof of this theorem in [27] based on an error in [26] However, this gap has been filled in [13] by the author. An example due to Draper and Keesling in [3] has shown that the hypothesis of finite-dimensionality cannot be eliminated in Theorem 1.2. In [18] Kozlowski and Segal show that a pointed metric continuum $(X, x)$ which is movable and which has $\pi_{k}(X, x)=0$ for all $k \geqslant 1$ must have trivial shape. So in case the space $Y$ or $X$ has the shape of a point, the dimension restrictions in Theorem 1.2 can be eliminated. The example described in $\S 3$ shows that a pointed movable continuum $(X, x)$ which is not metric may have $\pi_{k}(X, x)=0$ for all $k \geqslant 1$ with $(X, x)$ not having the shape of a point. It seems likely that the example in $\S 3$ will have further applications as well.

The second theorem that we state is a shape version of the Hurewicz theorem due to K. Kuperberg [19].

1.3. ThEOREM. Let $(X, x)$ be a pointed movable metric continuum. Suppose that $n \geqslant 2$ and that $\underline{\pi}_{k}(X, x)=0$ for $1 \leqslant k \leqslant n-1$. Then $H_{k}(X)=0$ for $1 \leqslant k \leqslant$ $n-1$ and $\underline{\pi}_{n}(X, x)$ is isomorphic to $H_{n}(X)$.

In $\S 4$ we show that the metrizability of $(X, x)$ cannot be eliminated without additional hypotheses. We also point out that a compact metric continuum described in [12] shows that movability is a necessary hypothesis even in the compact metric case.

The next theorem is due to Overton [28].

1.4. TheOREM. Let $(X, A)$ be a movable compact metric pair. Then the $\stackrel{\text { Cech }}{\text { Cech }}$ homology sequence of $(X, A)$ is exact.

Of course, the Čech homology sequence is not exact in general even for compact metric pairs. In $\$ 5$ we give an example of a movable compact Hausdorff pair with the Čech homology sequence of $(X, A)$ not exact. This answers a question posed in [28].

The next theorem is due to Keesling [10]. We first need a definition.

1.5. Definition. Let $H$ be an abelian group. Then $H$ is $\aleph_{1}$-free provided every countable subgroup of $H$ is free abelian. If $H$ is a torsion free abelian group, then $H$ is $\aleph_{1}$-free if and only if it has property $L$ (see [8] or [10] for the definition of property $L$ which is due to Pontryagin [28]). 
1.6. THEOREM. Let $(X, A)$ be a movable compact Hausdorff pair. Then $H^{n}(X, A) / \operatorname{Tor} H^{n}(X, A)$ is $\aleph_{1}$-free for all $n \geqslant 0$.

In [15] a more general result has been obtained which gives a Čech cohomological criterion for the movability and $n$-movability of an arbitrary topological space.

The last theorem we mention is due to Mardesić. However, the proof of Overton and Segal [29] is quite short.

1.7. THEOREM. Let $X$ be an n-dimensional $\mathrm{LC}^{n-1}$ peano continuum. Then $X$ is movable.

In [17] Kozlowski and Segal have shown that an $\mathrm{LC}^{n-1}$ paracompactum is uniformly movable in the sense of [17]. (Uniform movability in the sense of [17] implies movability in the sense of Definition 1.1. Thus the result of [17] implies Theorem 1.7 for $\mathrm{LC}^{n-1}$ paracompacta.) Combining Theorems 1.6 and 1.7 we have that every $n$-dimensional $\mathrm{LC}^{n-1}$ peano continuum $X$ has $H^{k}(X) / \operatorname{Tor} H^{k}(X)$ a free abelian group for all $k \geqslant 0$. However, in [15] a stronger result is shown. It is shown that if $X$ is any compact Hausdorff space which is $\mathrm{LC}^{n-1}$, then $H^{k}(X)$ is finitely generated for $0 \leqslant k \leqslant n-1$. Also in [15] results are obtained concerning the structure of the Čech cohomology of general paracompact spaces $X$ which are $\mathrm{LC}^{n-1}$. The results in [15] were used to give examples of movable compact metric continua $X$ which cannot be shape equivalent to any paracompact space $Y$ which is $\mathrm{LC}^{2}$.

2. Preliminaries. In this section we introduce some necessary preliminary results which will be used in the construction of the examples in $\S \S 3,4$, and 5 . We will need results concerning the shape of compact connected abelian topolog. ical groups obtained by the author in previous papers ([8], [9], [10], and [11]). An additional result will be needed concerning the Čech homology of compact connected abelian topological groups which will be proved in this section. Several results will be needed from the theory of abelian groups ([4] and [5]) which will be included in this section also. The first result is in [8] .

\subsection{THEOREM. Let $A$ and $B$ be compact connected abelian topological} groups. Then if $A$ and $B$ are shape equivalent, then they are isomorphic as topological groups.

In particular, two such topological groups which are shape equivalent are homeomorphic. It might seem that shape theory is trivial in the category of compact connected abelian topological groups because of Theorem 2.1. However, Theorem 2.1 allows us to translate shape invariant properties of spaces $A$ which are compact connected abelian topological groups into algebraic properties of their 
character groups, char $A$. Since $\operatorname{char} A \simeq H^{1}(A)$, this is equivalent to translating the shape invariant property into an algebraic property of $H^{1}(A)$. This allows us to use the theory of abelian groups to attack problems in shape theory. For example in [8] the shape invariant property of movability is translated into an algebraic property of $H^{1}(A)$ for $A$ a compact connected abelian topological group. This is the next theorem stated.

\subsection{THEOREM. Let $A$ be a compact connected abelian topological group.} Then $A$ is movable if and only if $H^{1}(A)$ is $\aleph_{1}$-free.

This is a partial converse to Theorem 1.6. Theorem 2.2 allows us to construct an arbitrary movable compact connected abelian topological group $A$ by taking an arbitrary $\aleph_{1}$-free abelian group $H$ and letting $A=\operatorname{char} H$. Then $A$ is movable since $H \simeq \operatorname{char} A \simeq H^{1}(A)$.

The next theorem is due to K. Hofmann [6] and shows how to compute the cohomology of a compact connected abelian topological group $A$ given $H^{1}(A)$.

\subsection{THEOREM. Let $A$ be a compact connected abelian topological group.} Then $H^{*}(A) \simeq \Lambda H^{1}(A) \simeq \Lambda$ char $A$.

Here $\Lambda G=\left\{\Lambda_{n} G\right\}$ of an abelian group $G$ is the exterior algebra of $G$. See $\S 6$ of Chapter XVI of [20] for the elementary properties of the exterior algebra.

2.4. THEOREM. Let $A$ be a compact connected abelian topological group. Then $H_{n}(A)$ is naturally isomorphic to $\operatorname{Hom}\left(H^{n}(A), Z\right)$.

Proof. Let $T^{k}$ be a $k$-dimensional torus. Then $H_{m}\left(T^{k}\right)$ is isomorphic to $Z^{k} C_{m}$ and thus is a finitely generated free abelian group for all $0 \leqslant m \leqslant k$ and is 0 for $m \geqslant k+1$. By the Universal Coefficient Theorem for singular cohomology [31, Theorem 3, p. 243] we have a functorial short exact sequence:

$$
0 \rightarrow \operatorname{Ext}\left(H_{n-1}\left(T^{k}\right), Z\right) \rightarrow H^{n}\left(T^{k}\right) \rightarrow \operatorname{Hom}\left(H_{n}\left(T^{k}\right), Z\right) \rightarrow 0 .
$$

Since $H_{n-1}\left(T^{k}\right)$ is a finitely generated free abelian group, $\operatorname{Ext}\left(H_{n-1}\left(T^{k}\right), Z\right)=0$. Thus there is a natural isomorphism between $H^{n}\left(T^{k}\right)$ and $\operatorname{Hom}\left(H_{n}\left(T^{k}\right), Z\right)$.

Let $H$ be a finitely generated free abelian group. We define the dual of $H$ to be $H^{*}=\operatorname{Hom}(H, Z)$. Clearly $H \simeq H^{*}=\operatorname{Hom}(H, Z)$, but not by a natural isomorphism. However, if one defines $h: H \rightarrow H^{* *}$ by $h(x)(g)=g(x)$ for $x \in H$ and $g \in H^{*}=\operatorname{Hom}(H, Z)$, then clearly $h$ is a natural isomorphism. Now $h$ induces the following natural isomorphism:

$$
H_{n}\left(T^{k}\right) \rightarrow \operatorname{Hom}\left(\operatorname{Hom}\left(H_{n}\left(T^{k}\right), Z\right), Z\right) .
$$

However, we have already shown that $H^{n}\left(T^{k}\right)$ is naturally isomorphic to Hom $\left(H_{n}\left(T^{k}\right), Z\right)$. Thus we have a natural isomorphism from $H_{n}\left(T^{k}\right)$ to $\operatorname{Hom}\left(H^{n}\left(T^{k}\right), Z\right)$ and the theorem is true for tori. 
Now let $A$ be an arbitrary compact connected abelian topological group. Let $A$ be the inverse limit of an inverse system of tori $\left\{T_{\alpha}^{k} ; \pi_{\alpha \beta} ; \alpha \leqslant \beta \in \Gamma\right\}$. Then there are the following natural isomorphisms:

$$
\begin{aligned}
H_{n}(A) & \simeq \underset{\lim }{\longleftarrow}\left\{H_{n}\left(T_{\alpha}^{k_{\alpha}}\right)\right\} \simeq \lim \left\{\operatorname{Hom}\left(H^{n}\left(T_{\alpha}^{k_{\alpha}}\right), Z\right)\right\} \\
& \simeq \operatorname{Hom}\left(\lim _{\longrightarrow}\left\{H^{n}\left(T_{\alpha}^{k_{\alpha}}\right)\right\}, Z\right) \simeq \operatorname{Hom}\left(H^{n}(A), Z\right) .
\end{aligned}
$$

This completes the proof of Theorem 2.4.

Theorem 2.3 and Theorem 2.4 allow us to algebraically compute the Čech cohomology and the Čech homology of a compact connected abelian topological group $A$ from the character group of $A$.

2.5. THEOREM. Let $A$ be a compact connected abelian topological group. Then there is a natural isomorphism $H_{1}(A) \simeq \underline{\pi}_{1}(A)$. Also $\underline{\pi}_{n}(A)=0$ for all $n>1$.

Proof. Let $T$ denote the circle group. Then by definition $\underline{\pi}_{1}(A)=$ $\operatorname{Mor}_{S C}(T, A)$ where $\operatorname{Mor}_{S C}(T, A)$ is given a natural group structure. By Theorem 1.2 of [8] the shape functor $S$ restricted to $\operatorname{Hom}(T, A)$ is one-to-one onto $\operatorname{Mor}_{S C}(T, A)$. One can also verify that $S: \operatorname{Hom}(T, A) \rightarrow \operatorname{Mor}_{S C}(T, A)=\underline{\pi}_{1}(A)$ is also a group homomorphism. Thus we have a natural isomorphism $\operatorname{Hom}(T, A)$ $\simeq \underline{\pi}_{1}(A)$. On the other hand $\operatorname{Hom}(T, A)$ is naturally isomorphic to

\section{Hom (char $A$, char $T$ )}

by the isomorphism which takes $g \in \operatorname{Hom}(T, A)$ to its dual homomorphism $g^{*} \in$ Hom (char $A$, char $T$ ). This gives us a natural isomorphism from $\operatorname{Hom}\left(H^{1}(A), Z\right)$ to $\underline{\pi}_{1}(A)$. Applying Theorem 2.4 we get the required natural isomorphism from $H_{1}(A)$ to $\underline{\pi}_{1}(A)$.

For the last part of the theorem let $A$ be the inverse limit of the inverse system of tori $\left\{T_{\alpha}^{k_{\alpha}} ; \pi_{\alpha \beta} ; \alpha \leqslant \beta \in \Gamma\right\}$. Then $\pi_{n}(A)$ is the inverse limit of the inverse system $\left\{\pi_{n}\left(T_{\alpha}^{k \alpha}\right) ; \pi_{\alpha \beta^{*}} ; \alpha \leqslant \beta \in \Gamma\right\}$. However, $\pi_{n}\left(T_{\alpha}^{k}\right)=0$ for all $n \geqslant 2$. Thus $\underline{\pi}_{n}(A)=0$ for all $n \geqslant 2$.

We denote the exterior algebra of an abelian group $H$ by $\Lambda H=\left\{\Lambda_{n} H\right\}$.

By definition $\Lambda H=T(H) / D\left(H^{2}\right)$ where $T(H)$ is the tensor algebra of $H$ [20, p. 558] and $D\left(H^{2}\right)$ is the ideal generated by all squares $h^{2}$ in $T(H)$ of elements $h$ in $H$. In particular, for each $n, \Lambda_{n}(H)$ is a quotient of the group $T_{n}(H)=H \otimes \cdots \otimes H$. Clearly if $\operatorname{Hom}(H, Z)=0$, then $\operatorname{Hom}\left(\bigotimes_{i=1}^{n} H, Z\right)=0$ also. Thus $\operatorname{Hom}\left(\Lambda_{n} H, Z\right)=0$ as well. Summarizing we have the following proposition.

2.6. Proposition. If $H$ is an abelian group with $\operatorname{Hom}(H, Z)=0$, then $\operatorname{Hom}\left(\Lambda_{n} H, Z\right)=0$ for all $n \geqslant 1$. 
In $\S 5$ we will need the following result which is due to E. C. Zeeman [5, Corollary 94.6, p. 162].

2.7. Theorem. Suppose that $\Gamma$ is a nonmeasurable set. Then there are natural isomorphisms:

$$
\operatorname{Hom}\left(\bigoplus_{\alpha \in \Gamma} Z, Z\right) \simeq \prod_{\alpha \in \Gamma} Z \text { and } \operatorname{Hom}\left(\prod_{\alpha \in \Gamma} Z, Z\right) \simeq \bigoplus_{\alpha \in \Gamma} Z \text {. }
$$

It is the latter natural isomorphism which is surprising and will be used in $\S 5$. The proof of Theorem 2.7 uses the concept of slenderness of an abelian group due to J. Łos' [5, p. 159] and the fact that the group of integers is slender [5, Proposition 94.2, p. 159].

Lastly we will need the fact that there exist $\aleph_{1}$-free abelian groups $H$ such that $\operatorname{Hom}(H, Z)=0$. A description of such an example can be found in [5, Exercise 13, p. 163].

2.8. THEOREM. There exist nontrivial $\aleph_{1}$-free abelian groups $H$ such that $\operatorname{Hom}(H, Z)=0$.

3. The Whitehead Theorem. Let $A$ be a compact connected abelian topological group. Then we first want to observe that $A$ is movable if and only if $(A, 0)$ is movable as a pointed compactum. This can be seen by modifying the proof of Theorem 2.5 of [8] using Theorem 1.2 of [8]. We leave this to the reader. In this section we describe a compact connected abelian topological group $A$ such that $A$ is movable with $H_{n}(A)=0$ for all $n \geqslant 1$ and $\underline{\pi}_{n}(A)=0$ for all $n \geqslant 1$, but with $A$ not shape equivalent to a point. If one lets $f:\{p\} \rightarrow A$ be defined by $f(p)=0$, then $f$ induces an isomorphism on all Čech homology groups and all shape groups, but is not a shape equivalence. In the example $A$ is not metrizable. According to [18] a movable metrizable pointed continuum $(X, x)$ with $\underline{\pi}_{n}(X, x)=0$ for all $n \geqslant 1$ must have trivial shape.

3.1. THEOREM. There is a movable compact connected abelian topological group $A$ such that $\underline{\pi}_{n}(A)=H_{n}(A)=0$ for all $n \geqslant 1$, but with $A$ not having the shape of a point.

Proof. Let $H$ be a nontrivial $\aleph_{1}$-free abelian group such that $\operatorname{Hom}(H, Z)$ $=0$. Such a group $H$ exists by Theorem 2.8. Let $A=\operatorname{char} H$. Then $A$ is a compact connected abelian topological group. By. Theorem $2.1, A$ is movable since $H^{1}(A) \simeq \operatorname{char} A \simeq H$ is $\aleph_{1}$-free. By Theorem 2.4, $H_{n}(A) \simeq \operatorname{Hom}\left(H^{n}(A), Z\right)$. By Proposition 2.6 together with Theorem 2.3, $H_{n}(A) \simeq \operatorname{Hom}\left(\Lambda_{n} H^{1}(A), Z\right) \simeq$ $\operatorname{Hom}\left(\Lambda_{n} H, Z\right)=0$ for all $n \geqslant 1$. By Theorem $2.5, \underline{\pi}_{n}(A)=0$ for all $n \geqslant 1$. Now $A$ does not have the shape of a point since $H^{1}(A) \simeq H$ is nontrivial. This proves the theorem.

In [18] Kozlowski and Segal point out that the compact connected abelian 
topological group described in Theorem 3.1 (which was first mentioned by the author in [14]) is an example of a movable continuum which is not uniformly movable.

4. The Hurewicz theorem. In [19] $\mathrm{K}$. Kuperberg showed that if $(X, x)$ is a pointed movable metric continuum with $\underline{\pi}_{k}(X, x)=0$ for $1 \leqslant k \leqslant n-1$ with $n \geqslant 2$, then $H_{k}(X)=0$ for $1 \leqslant k \leqslant n-1$ and $\underline{\pi}_{n}(X, x)$ is isomorphic to $H_{n}(X)$. In this section we show that this may be false if $X$ is not metric.

Before we present the main result of this section we present an example described in [12] which shows that movability cannot be eliminated in Theorem 1.3 without additional hypotheses even for metric continua. The following was proved in [12].

4.1. THEOREM. Let $(Z, z)$ be a pointed metric continuum. Then there is a metric continuum $X$ with $a, b \in X$ such that, for all $n \geqslant 1, \underline{\pi}_{n}(X, a) \simeq$ $\underline{\pi}_{n}(Z, z)$ and $\underline{\pi}_{n}(X, b)=0$.

The example which proves Theorem 4.1 is described in [12] as the wedge of the dyadic solenoid $\Sigma_{2}$ and the space $Z$. The point $a$ is the wedge point and $b$ is a point in $\Sigma_{2}$ not in the same arc component as $a$. This is the basis of our next example.

4.2. EXAmple. Let $(Z, z)$ be a pointed connected finite polyhedron with $\pi_{k}(Z, z)=0$ for $1 \leqslant k \leqslant n-1$ and $\pi_{n}(Z, z) \neq 0$ where $n \geqslant 2$ is any integer.

Now whatever the choice of base point $H_{k}\left(Z \vee \Sigma_{2}\right) \simeq H_{k}(Z) \oplus H_{k}\left(\Sigma_{2}\right) \simeq H_{k}(Z)$ which will be zero for $1 \leqslant k \leqslant n-1$ and nonzero for $k=n$. Now for the base point $a$ described above, $\underline{\pi}_{k}\left(Z \vee \Sigma_{2}, a\right) \simeq \pi_{k}(Z, z) \simeq H_{k}(Z)=H_{k}\left(Z \vee \Sigma_{2}\right)$ and there is no contradiction to the Hurewicz theorem. However, for the base point $b$ described above, $\underline{\pi}_{k}\left(Z \vee \Sigma_{2}, b\right)=0$ for all $k$. Thus for $k=n, \underline{\pi}_{n}\left(Z \vee \Sigma_{2}, b\right)$ $\neq H_{n}\left(Z \vee \Sigma_{2}\right)$. Of course, the example here is not movable.

In the remainder of this section we describe two movable pointed continua $\left(X_{n}, x_{n}\right)$ and $\left(Y_{n}, y_{n}\right)$ such that $\underline{\pi}_{k}\left(X_{n}, x_{n}\right)=\underline{\pi}_{k}\left(Y_{n}, y_{n}\right)=0$ for $1 \leqslant k \leqslant n-1$ and $H_{k}\left(X_{n}\right)=H_{k}\left(Y_{n}\right)=0$ for $1 \leqslant k \leqslant n-1$. However, either $H_{n}\left(X_{n}\right) \neq$ $\underline{\pi}_{k}\left(X_{n}, x_{n}\right)$ or $H_{n}\left(Y_{n}\right) \neq \underline{\pi}_{n}\left(Y_{n}, y_{n}\right)$. Thus either $X_{n}$ or $Y_{n}$ is a counterexample to the nonmetric version of Theorem 1.3.

4.3. THEOREM. Let $n \geqslant 2$ be given. There is a nonmetric movable pointed continuum $\left(Z_{n}, z_{n}\right)$ such that for $1 \leqslant k \leqslant n-1, H_{k}\left(Z_{n}\right)=\underline{\pi}_{k}\left(Z_{n}, z_{n}\right)=0$ and $\underline{\pi}_{n}\left(Z_{n}\left(Z_{n}, z_{n}\right)\right) \simeq H_{n}\left(Z_{n}\right)$.

Proof. Let $n \geqslant 2$ be given. We will describe two pointed movable continua $\left(X_{n}, x_{n}\right)$ and $\left(Y_{n}, y_{n}\right)$ and then show that at least one of them satisfies the conditions of Theorem 4.3. We cannot at this time be certain which of the two examples is in fact the counterexample we seek, but at least one of them is a 
counterexample. Both $X_{n}$ and $Y_{n}$ are homeomorphic, but the choice of base points in each is different. We now describe $\left(X_{n}, x_{n}\right)$ and $\left(Y_{n}, y_{n}\right)$.

Let $H$ be a nontrivial $\aleph_{1}$-free abelian group with $\operatorname{Hom}(H, Z)=0$. Let $A=$ char $H$. Then as in the previous section $A$ is movable and $\pi_{k}(A)=0$ for all $k \geqslant 1$ and $H_{k}(A)=0$ for all $k \geqslant 1$. Now let $f: A \rightarrow A$ be defined by $f(x)=$ $2 x$. Then $f$ is a continuous homomorphism. Note that the dual homomorphism $f^{*}: \operatorname{char} A \rightarrow \operatorname{char} A$ is also defined by $f^{*}(x)=2 x$. Since char $A \simeq H$ is $\aleph_{1}$-free and thus torsion free, $f^{*}$ must be one-to-one. Thus $f$ must be onto by duality. We now claim that $f$ is not one-to-one.

Claim 1. $f: A \rightarrow A$ is not one-to-one.

Proof of Claim 1. We will show this by showing $f^{*}$ is not onto. Now $f^{*}: H \rightarrow H$ is defined by $f^{*}(x)=2 x$. If $f^{*}$ were onto, then letting $x \neq 0$ in $H$ we would have that for every $n$, there is a $y \in H$ such that $2^{n} y=x$. Since $H$ is torsion free, these roots are unique. Thus we get a copy of the group $Q^{(2)}=$ $\left\{m / 2^{n}: m, n \in Z\right\}$ contained in $H$. However, $Q^{(2)}$ is a countable subgroup of $H$ which is not free, contradicting the fact that $H$ is $\aleph_{1}$-free. Thus $f^{*}$ cannot be onto and $f$ cannot be one-to-one.

Since $f$ is not one-to-one, $\operatorname{ker} f \neq 0$. Let $y \in A$ be such that $y \neq 0$ with $2 y=0$. Now let $X_{n}=A \vee S^{n}=Y_{n}$ where the wedge point is $0 \in A$. Let the base point $x_{n}$ in $X_{n}$ be the wedge point and let the base point $y_{n} \in Y_{n}$ be $y_{n}=y$.

ClaIM 2. $\left(X_{n}, x_{n}\right)$ is movable as a pointed continuum.

Proof of Claim 2. Whenever one takes the wedge of two pointed movable continua where the wedge points are the base points and the base point of the wedge is the wedge point, then the resultant pointed continuum is movable. This is routine and we leave the details to the reader. However, if the base point chosen is not the wedge point as in the case of $\left(Y_{n}, y_{n}\right)$, then the movability is more difficult.

Claim 3. $\left(Y_{n}, y_{n}\right)$ is movable as a pointed continuum.

Before we prove Claim 3 we will need the following technical lemma which we state as Claim 4.

ClAIM 4. Let $B$ be a compact connected abelian topological group. If $B$ is movable, then there is an inverse system of tori $\left\{T_{\alpha}^{n_{\alpha}} ; \pi_{\alpha \beta} ; \alpha \leqslant \beta \in \Gamma\right\}$ with continuous onto homomorphisms as bonding maps having $B$ as inverse limit such that whenever $\beta \geqslant \alpha$ there is a continuous homomorphism $r^{\alpha \beta}: T_{\alpha}^{n_{\alpha}} \rightarrow T_{\beta}^{n_{\beta}}$ such that $\pi_{\alpha \beta} \circ r^{\alpha \beta}$ is the identity on $T_{\alpha}^{n_{\alpha}}$. Furthermore, $T_{\beta}^{n_{\beta}} \simeq T_{\alpha}^{n_{\alpha}} \times T^{m}$ with the $\pi_{\alpha \beta}: T_{\beta}^{n_{\beta}} \rightarrow T_{\alpha}^{n_{\alpha}}$ equivalent to the projection map of $T_{\alpha}^{n_{\alpha}} \times T^{m}$ onto $T_{\alpha}^{n_{\alpha}}$.

Proof of Claim 4. The proof uses Pontryagin duality. Let $H=\operatorname{char} B$. Then $H$ is $\aleph_{1}$-free since $B$ is movable by Theorem 1.6. A pure subgroup $G$ of $H$ is one such that $H / G$ is torsion free. Recall that $H$ is $\aleph_{1}$-free if and only if 
every finitely generated subgroup $G$ is contained in a finitely generated pure subgroup of $H$. As is well known, $H$ is the direct limit of its finitely generated subgroups directed by inclusion. Thus $H$ is also the direct limit of its finitely generated pure subgroups since it is $\aleph_{1}$-free and the finitely generated pure subgroups of $H$ are cofinal in the direct system of all finitely generated subgroups. Let $\left\{G_{\alpha} ; e_{\alpha \beta} ; \alpha \leqslant \beta \in \Gamma\right\}$ be the directed system of all finitely generated pure subgroups of $H$. Then let $T_{\alpha}^{n_{\alpha}}=\operatorname{char} G_{\alpha}$ and $\pi_{\alpha \beta}=e_{\alpha \beta}^{*}$ be the dual homomorphism for $\alpha \leqslant \beta$. By duality and the fact that $H$ is the direct limit of $\left\{G_{\alpha}\right\}$, we have that $B$ is the inverse limit of the inverse system $\left\{T_{\alpha}^{n_{\alpha}} ; \pi_{\alpha \beta} ; \alpha \leqslant \beta \in \Gamma\right\}$. We now show that this inverse system has the properties required in Claim 4. The bonding maps are onto homomorphisms since the $e_{\alpha \beta}$ 's are one-to-one. Now let $\beta \geqslant \alpha$. Then since $G_{\alpha}$ is a pure subgroup of $H$, it is also a pure subgroup of $G_{\beta} \supset G_{\alpha}$. Thus $G_{\beta} / G_{\alpha}$ is torsion free. Since $G_{\beta} / G_{\alpha}$ is finitely generated, $G_{\beta} / G_{\alpha}$ is a finitely generated torsion free abelian group and thus isomorphic to some $Z^{m}$. This implies that $G_{\beta} \simeq Z^{m} \oplus G_{\alpha}$. Thus there is a homomorphism $g: G_{\beta} \rightarrow G_{\alpha}$ such that $g \circ e_{\alpha \beta}=1_{G_{\alpha}}$. Let $r^{\alpha \beta}=g^{*}$ be the dual homomorphism. Then $\pi_{\alpha \beta} \circ r^{\alpha \beta}$ is the identity on $T_{\alpha}^{n_{\alpha}}$. Note that the kernel of $g^{*}$ will be isomorphic to char $Z^{m}$ $=T^{m}$ and that $T_{\beta}^{n \beta}$ will be isomorphic to $T_{\alpha}^{n} \times T^{m}$ with $\pi_{\alpha \beta}$ equivalent to the projection onto $T_{\alpha}^{n_{\alpha}}$.

ProOF OF ClaIM 3. Let $\left\{T_{\alpha}^{n} ; \pi_{\alpha \beta} ; \alpha \leqslant \beta \in \Gamma\right\}$ be an inverse system of tori having the group $A$ as inverse limit such that $\left.\left\{T_{\alpha}^{n}\right\}\right\}$ has the properties given in Claim 4. Let $y_{\alpha} \in T_{\alpha}^{n_{\alpha}}$ be the image of $y \in A$ under the projection maps $\pi_{\alpha}: A \rightarrow T_{\alpha}^{n_{\alpha}}$ making $A$ the inverse limit. Let $Z_{\alpha}=T_{\alpha}^{n_{\alpha}} \vee S^{n}$ for all $\alpha$ where the wedge point is the identity element $0_{\alpha} \in T_{\alpha}^{n_{\alpha}}$. Now for all $\alpha$ in a cofinal $\Gamma^{\prime} \subset \Gamma, \pi_{\alpha}(y)=y_{\alpha} \neq \pi_{\alpha}(0)=0_{\alpha}$ since $y \neq 0$. Assume therefore that the inverse system has the property that $y_{\alpha} \neq 0_{\alpha}$ for all $\alpha \in \Gamma$ by replacing $\Gamma$ by $\Gamma^{\prime}$. Now let $f_{\alpha \beta}: Z_{\beta} \rightarrow Z_{\alpha}$ be defined by $f_{\alpha \beta}=\pi_{\alpha \beta} \vee 1_{s^{n}}$. Then the inverse system $\left\{Z_{\alpha} ; f_{\alpha \beta} ; \alpha \leqslant \beta \in \Gamma\right\}$ has $A \vee S^{n}$ as its inverse limit. Also $\left\{\left(Z_{\alpha}, y_{\alpha}\right) ; f_{\alpha \beta}\right.$; $\alpha \leqslant \beta \in \Gamma\}$ has $\left(Y_{n}, y_{n}\right)$ as its inverse limit. Let $\beta \geqslant \alpha$ in $\Gamma$. We now show that there is a map $r^{\alpha \beta}:\left(Z_{\alpha}, y_{\alpha}\right) \rightarrow\left(Z_{\beta}, y_{\beta}\right)$ such that $f_{\alpha \beta} \circ r^{\alpha \beta}:\left(Z_{\alpha}, y_{\alpha}\right) \rightarrow$ $\left(Z_{\alpha}, y_{\alpha}\right)$ is the identity. This will clearly imply that the system $\left\{\left(Z_{\alpha}, y_{\alpha}\right)\right\}$ is movable and thus that $\left(Y_{n}, y_{n}\right)$ is movable as asserted. We will show the existence of $r^{\alpha \beta}$ in Claim 5.

CLAIM 5. Let $\beta \geqslant \alpha \in \Gamma$. Then there is a map $r^{\alpha \beta}:\left(Z_{\alpha}, y_{\alpha}\right) \rightarrow\left(Z_{\beta}, y_{\beta}\right)$ such that $f_{\alpha \beta} \circ r^{\alpha \beta}=1\left(z_{\alpha}, y_{\alpha}\right)$.

Proof of Claim 5. Let $g: T_{\alpha}^{n_{\alpha}} \rightarrow T_{\beta}^{n_{\beta}}$ be the continuous homomorphism given by Claim 4 such that $\pi_{\alpha \beta} \circ g$ is the identity on $T_{\alpha}^{n} \alpha$ and with the image $g\left(T_{\alpha}^{n_{\alpha}}\right)$ of $g$ in $T_{\beta}^{n} \beta_{\text {a factor of }} T_{\beta}^{n}$. Let the map $h: Z_{\alpha} \rightarrow Z_{\beta}$ be defined by $h=g \vee 1_{s^{n}}$. Then $f_{\alpha \beta} \circ h$ is the identity on $Z_{\alpha}$. However, $h$ is not a map from 
$\left(Z_{\alpha}, y_{\alpha}\right) \rightarrow\left(Z_{\beta}, y_{\beta}\right)$ since $h$ may not take $y_{\alpha}$ to $y_{\beta}$. We need to modify $h$ to get the map $r^{\alpha \beta}$ which is required. From Claim $4, T_{\beta}^{n \beta} \simeq T_{\alpha}^{n} \times T^{m}$ with the map $\pi$ equivalent to the projection map onto $T_{\alpha}^{n_{\alpha}}$. Also $g: T_{\alpha}^{n_{\alpha}} \rightarrow T_{\beta}^{n_{\beta}}$ is equivalent to the map taking $T_{\alpha}^{n_{\alpha}}$ isomorphically onto $T_{\alpha}^{n_{\alpha}} \times\{0\} \subset T_{\alpha}^{n_{\alpha}} \times T^{m}$. Then $y_{\beta}$ will correspond to some $\left(y_{\alpha}, b\right)$ for some $b \in T^{m}$. Using the fact that $T^{m}$ is a manifold one can get a homotopy $p_{t}: T^{m} \rightarrow T^{m}$ for $0 \leqslant t \leqslant 1$ such that $p_{0}$ is the identity on $T^{m}$ and $p_{1}(0)=b$. Now let $U$ be an open set in $T_{\alpha}^{n_{\alpha}}$ containing $y_{\alpha}$ such that $0_{\alpha} \notin U$ and let $q: T_{\alpha}^{n_{\alpha}} \rightarrow[0,1]$ be a Urysohn function with $q\left(y_{\alpha}\right)=1$ and $q \mid T_{\alpha}^{n_{\alpha}}-U \equiv 0$, Then let $g^{\prime}: T_{\alpha}^{n_{\alpha}} \rightarrow T_{\alpha}^{n_{\alpha}} \times T^{m}=T_{\beta}^{n_{\beta}}$ be defined by $g^{\prime}(x)=\left(x, p_{q(x)} \circ \pi_{2} \circ g(x)\right)$ where $\pi_{2}: T_{\alpha}^{n_{\alpha}} \times T^{m} \rightarrow T^{m}$ is the projection. Then $g^{\prime}(0)=0$ and $g^{\prime}\left(y_{\alpha}\right)=y_{\beta}$. Then $r^{\alpha \beta}=g^{\prime} \vee 1_{s^{n}}$ will be well defined and $r^{\alpha \beta}\left(y_{\alpha}\right)=y_{\beta}$ with $f_{\alpha \beta} \circ r^{\alpha \beta}=1\left(z_{\left.\alpha, y_{\alpha}\right)}\right.$ as asserted. The map $r^{\alpha \beta}$ is simply a "twisting" of the map $h$ described above so that $r^{\alpha \beta}$ takes $y_{\alpha}$ to $y_{\beta}$.

We now resume the proof of Theorem 4.3. We now know that both $\left(X_{n}, x_{n}\right)$ and $\left(Y_{n}, y_{n}\right)$ are movable as pointed continua. It is also clear that $H_{k}\left(X_{n}\right)=H_{k}\left(Y_{n}\right)=H_{k}\left(S^{n}\right)$ for all $k \geqslant 0$. Thus $H_{k}\left(X_{n}\right)=H_{k}\left(Y_{n}\right)=0$ for $1 \leqslant$ $k \leqslant n-1$ and $H_{n}\left(X_{n}\right)=H_{n}\left(Y_{n}\right)=Z$.

Claim 6. $\underline{\pi}_{k}\left(X_{n}, x_{n}\right)=\underline{\pi}_{k}\left(Y_{n}, y_{n}\right)=0$ for $1 \leqslant k \leqslant n-1$.

Proof of Claim 6. Let $A$ be the inverse limit of the inverse system of tori $\left\{T_{\alpha}^{n_{\alpha}} ; \pi_{\alpha \beta} ; \alpha \leqslant \beta \in \Gamma\right\}$ and let $Z_{\alpha}=T_{\alpha}^{n \alpha} \vee S^{n}$ and the inverse system $\left\{Z_{\alpha} ; f_{\alpha \beta} ; \alpha \leqslant \beta \in \Gamma\right\}$ be defined as in the proof of Claim 5. Let $x_{\alpha} \in Z_{\alpha}$ and $y_{\alpha} \in Z_{\alpha}$ be such that $\left(X_{n}, x_{n}\right)$ and $\left(Y_{n}, y_{n}\right)$ are the inverse limits of the inverse systems $\left\{\left(Z_{\alpha}, x_{\alpha}\right)\right\}$ and $\left\{\left(Z_{\alpha}, y_{\alpha}\right)\right\}$, respectively. Then the inclusion maps $e_{\alpha}$ : $T_{\alpha}^{n_{\alpha}} \rightarrow Z_{\alpha}=T_{\alpha}^{n_{\alpha}} \vee S^{n}$ induce isomorphisms of the homotopy groups of $T_{\alpha}^{n_{\alpha}}$ onto those of $Z_{\alpha}$ for $1 \leqslant k \leqslant n-1$ so that for all $\beta \geqslant \alpha$ the following diagram commutes for $1 \leqslant k \leqslant n-1$.

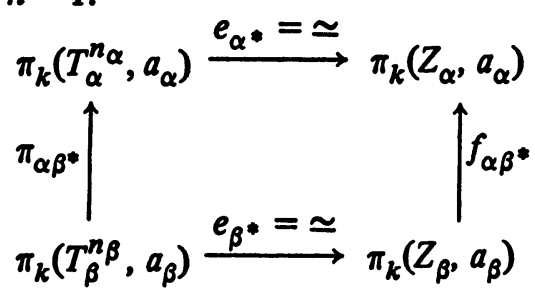

Here $a_{\alpha}=x_{\alpha}$ or $a_{\alpha}=Y_{\alpha}$, respectively. Thus for $1 \leqslant k \leqslant n-1$ the inverse system of groups $\left\{\pi_{k}\left(T_{\alpha}^{n}, a_{\alpha}\right)\right\}$ is equivalent to the inverse system $\left\{\pi_{k}\left(Z_{\alpha}, a_{\alpha}\right)\right\}$. The first system has $\underline{\pi}_{k}\left(A, a_{\alpha}\right)$ as its limit and the second has $\underline{\pi}_{k}\left(X_{n}, x_{n}\right)$ or $\pi_{k}\left(Y_{n}, y_{n}\right)$ as limit depending on whether $a_{\alpha}=x_{\alpha}$ or $a_{\alpha}=y_{\alpha}$. Recall that $A=$ char $H$ where $H$ is $\aleph_{1}$-free and $\operatorname{Hom}(H, Z)=0$. Thus by the proof of Theorem 3.1, $\underline{\pi}_{k}\left(A, a_{\alpha}\right)=0$ for all $k \geqslant 1$. Thus, $\underline{\pi}_{k}\left(X_{n}, x_{n}\right)=\underline{\pi}_{k}\left(Y_{n}, y_{n}\right)=$ 0 for $1 \leqslant k \leqslant n-1$.

Resuming the proof of Theorem 4.3, all that remains to be shown is that either $\underline{\pi}_{n}\left(X_{n}, x_{n}\right) \neq Z$ or $\underline{\pi}_{n}\left(Y_{n}, y_{n}\right) \neq Z$. This will imply that one of these 
spaces is the $\left(Z_{n}, z_{n}\right)$ which will satisfy the conclusion of Theorem 4.3. This is the most difficult part of the proof and will be given as Claim 7 .

ClaIm 7. Either $\underline{\pi}_{n}\left(X_{n}, x_{n}\right) \neq Z$ or $\underline{\pi}_{n}\left(Y_{n}, y_{n}\right) \neq Z$.

Proof of Claim 7. The proof will be by contradiction. Suppose that $\underline{\pi}_{n}\left(X_{n}, x_{n}\right)=\underline{\pi}_{n}\left(Y_{n}, y_{n}\right)=Z$. Now let $A$ be the inverse limit of the system of tori $\left\{T_{\alpha}^{n_{\alpha}} ; \pi_{\alpha \beta} ; \alpha \leqslant \beta \in \Gamma\right\}$ and $\left(X_{n}, x_{n}\right)$ and $\left(Y_{n}, y_{n}\right)$ be the inverse limit of the inverse system $\left\{\left(Z_{\alpha^{\prime}}, a_{\alpha}\right) ; f_{\alpha \beta} ; \alpha \leqslant \beta \in \Gamma\right\}$ for $a_{\alpha}=x_{\alpha}$ and $a_{\alpha}=y_{\alpha^{\prime}}$ respectively, where $Z_{\alpha}=T_{\alpha}^{n_{\alpha}} \vee S^{n}$. This is as in the proof of Claim 6. Then the groups $\underline{\pi}_{n}\left(X_{n}, x_{n}\right)$ and $\underline{\pi}_{n}\left(Y_{n}, y_{n}\right)$ are the inverse limits of the inverse systems $\left\{\pi_{n}\left(Z_{\alpha}, a_{\alpha}\right) ; f_{\alpha \beta^{*}} ; \alpha \leqslant \bar{\beta} \in \Gamma\right\}$ for $a_{\alpha}=x_{\alpha}$ and $a_{\alpha}=y_{\alpha^{\prime}}$ respectively. Thus each of these inverse systems have the integers $Z$ as their inverse limit since we are assuming $\underline{\pi}_{n}\left(X_{n}, x_{n}\right)=\underline{\pi}_{n}\left(Y_{n}, y_{n}\right)=Z$. Now if $\left(\tilde{Z}_{\alpha}, \widetilde{a}_{\alpha}\right)$ is the universal covering space of $\left(Z_{\alpha}, a_{\alpha}\right)$, then $\widetilde{Z}_{\alpha}$ is homeomorphic to $R^{n_{\alpha}}$ with an $S^{n}$ attached at each of the points $Z^{n_{\alpha}} \subset R^{n_{\alpha}}$. We let the base points $\widetilde{a}_{\alpha}$ be $0 \in R^{n_{\alpha}}$ for $\widetilde{a}_{\alpha}=$ $\tilde{x}_{\alpha}$ and for $\tilde{a}_{\alpha}=\tilde{y}_{\alpha}$ let $\tilde{a}_{\alpha}=\tilde{y}_{\alpha} \in R^{n}$ be ary point such that $\tau_{\alpha}\left(\tilde{y}_{\alpha}\right)=y_{\alpha}$ where $\tau_{\alpha}: R^{n_{\alpha}} \rightarrow T_{\alpha}^{n_{\alpha}}$ is the covering map. Also let $\tau_{\alpha}$ denote the covering map from $\widetilde{Z}_{\alpha}$ to $Z_{\alpha}$. Note that $2 \widetilde{y}_{\alpha} \in Z^{n_{\alpha}}$ since $2 y_{\alpha}=0$. Now since $n \geqslant 2, \tau_{\alpha^{*}}$ :

$\pi_{n}\left(\widetilde{Z}_{\alpha}, \widetilde{a}_{\alpha}\right) \rightarrow \pi_{n}\left(Z_{\alpha}, a_{\alpha}\right)$ is an isomorphism. Also for $\beta \geqslant \alpha$, there are maps $\widetilde{f}_{\alpha \beta}:\left(\widetilde{Z}_{\beta}, \widetilde{a}_{\beta}\right) \rightarrow\left(\widetilde{Z}_{\alpha}, \widetilde{a}_{\alpha}\right)$ such that the following diagram commutes.

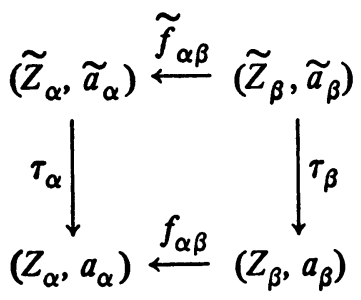

Thus for $\beta \geqslant \alpha$, we have the following:

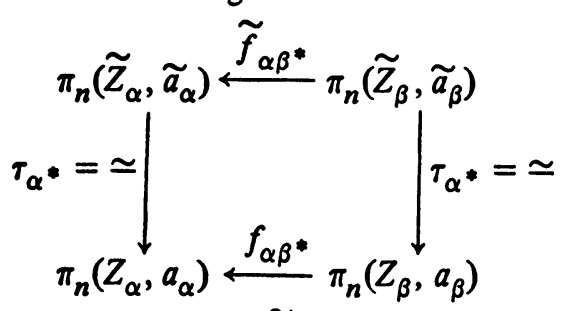

Thus the systems $\left\{\pi_{n}\left(Z_{\alpha}, a_{\alpha}\right)\right\}$ and $\left\{\pi_{n}\left(\tilde{Z}_{\alpha}, \tilde{a}_{\alpha}\right)\right\}$ are equivalent for $a_{\alpha}=x_{\alpha}$ and $a_{\alpha}=y_{\alpha}$. Thus the integers $Z$ must also be the inverse limit of the systems $\left\{\pi_{n}\left(\tilde{Z}_{\alpha}, \tilde{a}_{\alpha}\right)\right\}$ for $\tilde{a}_{\alpha}=\tilde{x}_{\alpha}$ and $\tilde{a}_{\alpha}=\tilde{y}_{\alpha}$. Let us now consider the inverse system of groups for $\tilde{a}_{\alpha}=\tilde{x}_{\alpha}$. Now the group $H_{\alpha}=\pi_{n}\left(\tilde{Z}_{\alpha}, \tilde{x}_{\alpha}\right)$ is clearly isomorphic to $\bigoplus_{i=1}^{\infty} Z$, the free abelian group with countably many generators. Each $S^{n}$ attached in $\widetilde{Z}_{\alpha}$ serves as one of the free generators for $H_{\alpha}$. Consider the $S^{n}$ attached at $\widetilde{x}_{\alpha}=0 \in R^{n_{\alpha}}$. Then for each $\alpha \in \Gamma$ there is induced a one-to-one homomorphism $h_{\alpha}: \pi_{n}\left(S^{n}, *\right) \rightarrow H_{\alpha}$ such that for all $\beta \geqslant \alpha$, we have: 


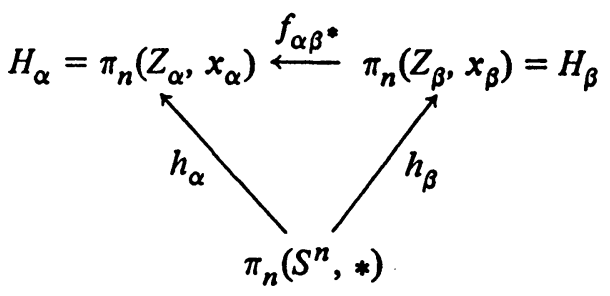

Thus there is a unique induced homomorphism $h$ from $\pi_{n}\left(S^{n}, *\right)$ into $Z$ as the inverse limit of $\left\{H_{\alpha}\right\}$. The homomorphism $h$ is one-to-one since each $h_{\alpha}$ is oneto-one. Suppose that $h\left(\pi_{n}\left(S^{n}, *\right)\right) \neq Z$. Let $c$ be an element in the inverse limit of the $H_{\alpha}$ 's with $c$ not in the image of $h$. Let $c_{\alpha} \in H_{\alpha}$ be the projection of $c$ into each $H_{\alpha}$. Let $m \in Z^{n_{\alpha}}$ and let $h_{m}$ be the generator in $H_{\alpha}$ corresponding to the $S^{n}$ attached at the point $m \in R^{n_{\alpha}}$. Then let $\left\{n_{\alpha}(m)\right\}$ be the coefficients of $c_{\alpha}$ so that $c_{\alpha}=\Sigma n_{\alpha}(m) h_{m}$. If for each $\alpha \in \Gamma, n_{\alpha}(m)=0$ for all $m \neq 0$, then $n_{\alpha}(0)=n_{\beta}(0)$ for all $\alpha$ and $\beta$ in $\Gamma$. However, this clearly implies that $c$ is in the image of $h$, a contradiction. Thus we conclude that there must be an $\alpha \in \Gamma$ such that $n_{\alpha}(m) \neq 0$ for some $m \neq 0$. However, for this $\alpha$, the projection of the inverse limit group $Z$ into $H_{\alpha}$ contains the group generated by $h_{\alpha}\left(\pi_{n}\left(S^{n}, *\right)\right) \cup\left\{c_{\alpha}\right\}$. Since $c_{\alpha}$ is linearly independent of the group $h_{\alpha}\left(\pi_{n}\left(S^{n}, *\right)\right)$, this implies that the projection of $Z$ into $H_{\alpha}$ has rank greater than or equal to 2 which is not possible. This contradiction shows that the homomorphism $h$ must be onto. We thus consider $Z=\pi_{n}\left(S^{n}, *\right)$ as the inverse limit of $\left\{\pi_{n}\left(\tilde{Z}_{\alpha}, \tilde{x}_{\alpha}\right)\right.$; $\left.\widetilde{f}_{\alpha \beta^{*}} ; \alpha \leqslant \beta \in \Gamma\right\}$ with the $h_{\alpha}$ 's the projection maps making $Z=\pi_{n}\left(S^{n}, *\right)$ the inverse limit.

On the other hand, $Z$ is also the inverse limit of the inverse system of groups $\left\{\pi_{n}\left(\tilde{Z}_{\alpha}, \tilde{y}_{\alpha}\right) ; \widetilde{f}_{\alpha \beta^{*}} ; \alpha \leqslant \beta \in \Gamma\right\}$. Let the projection homomorphisms be denoted by $p_{\alpha}: Z \rightarrow \pi_{n}\left(\widetilde{Z}_{\alpha}, \widetilde{y}_{\alpha}\right)$. Earlier we defined the map $f: A \rightarrow A$ by $f(x)=2 x$. Let $g:\left(Y_{n}, y_{n}\right) \rightarrow\left(X_{n}, x_{n}\right)$ be defined by $g(x)=f(x)$ for $x \in A \subset$ $Y_{n}=A \vee S^{n}$ and $g(x)=x$ for $x \in S^{n} \subset Y_{n}$. Then $g$ is well defined since $f(0)=0$. Also $g(y)=0$ since $2 y=0$ and thus $g\left(y_{n}\right)=x_{n}$ and $g$ is base point preserving. Clearly $g$ is continuous. We define $g_{\alpha}:\left(Z_{\alpha}, y_{\alpha}\right) \rightarrow\left(Z_{\alpha}, x_{\alpha}\right)$ by $g_{\alpha}(x)=2 x$ for $x \in T_{\alpha}^{n_{\alpha}}$ and $g_{\alpha}(x)=x$ for $x \in S^{n}$. Then for all $\beta \geqslant \alpha$, the following diagram commutes.

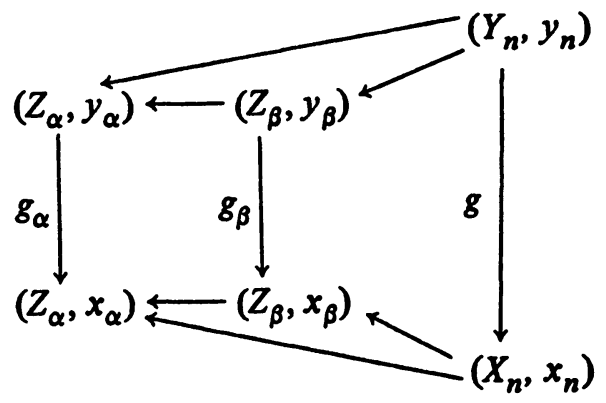


The $g_{\alpha}$ 's also induce maps $\widetilde{g}_{\alpha}:\left(\tilde{Z}_{\alpha}, \widetilde{y}_{\alpha}\right) \rightarrow\left(\tilde{Z}_{\alpha}, \widetilde{x}_{\alpha}\right)$ so that for all $\beta \geqslant \alpha$, the following diagram commutes.

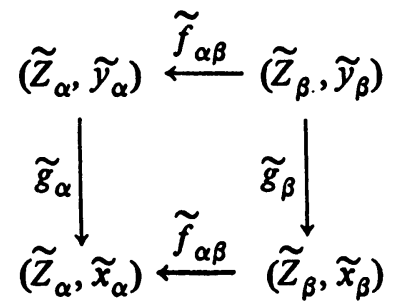

Thus for all $\beta \geqslant \alpha$ we have:

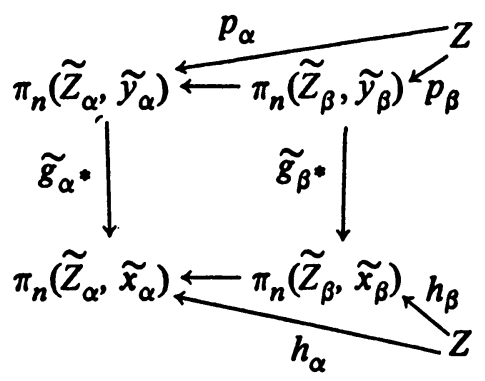

Thus there is a unique homomorphism $\phi: Z \rightarrow Z$ making the above diagram commute for all $\alpha, \beta \in \Gamma$. Now we will show that (1) $\widetilde{g}_{\alpha}$ is one-to-one for all

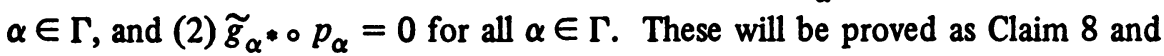
Claim 9. We will now show how (1) and (2) complete the proof of Claim 7. If (1) and (2) hold, then $\phi$ must be one-to-one since $\widetilde{g}_{\alpha}$ is one-to-one for all $\alpha$ by (1). But by (2), $\phi$ would have to be the zero homomorphism, a contradiction. This contradiction shows that either $\underline{\pi}_{n}\left(X_{n}, x_{n}\right) \neq Z$ or $\underline{\pi}_{n}\left(Y_{n}, y_{n}\right) \neq Z$ and Claim 7 is proved. Claim 7 completes the proof of Theorem 4.3. Thus our proof will be finished once we have shown Claim 8 and Claim 9.

Claim 8. $\widetilde{g}_{\alpha}$ is one-to-one for all $\alpha \in \Gamma$.

Proof of CLAIM 8. Note that $\widetilde{g}_{\alpha}:\left(\tilde{Z}_{\alpha}, \tilde{y}_{\alpha}\right) \rightarrow\left(\tilde{Z}_{\alpha}, \tilde{x}_{\alpha}\right)$ can be described as an imbedding. The mapping $\tilde{g}_{\alpha}$ takes $\tilde{y}_{\alpha}$ to $\tilde{x}_{\alpha}$ and $\widetilde{g}_{\alpha} \mid R^{n_{\alpha}}$ is a translation of $\tilde{y}_{\alpha}$ to $\tilde{x}_{\alpha}=0$ followed by the map $2 x: R^{n_{\alpha}} \rightarrow R^{n_{\alpha}}$. The points at which the $S^{n}$ 's are attached in $\left(Z_{\alpha}, y_{\alpha}\right)$ will be taken into the integer points under $g_{\alpha}$ since $2 \tilde{y}_{\alpha} \in Z^{n_{\alpha}} \subset R^{n_{\alpha}}$. Then $\widetilde{g}_{\alpha}$ will be the identity on the respective $S^{n}$ 's. Now $\widetilde{\mathrm{g}}_{\alpha}$ * will be one-to-one from $\pi_{n}\left(\widetilde{Z}_{\alpha}, \tilde{y}_{\alpha}\right)$ into $\pi_{n}\left(\widetilde{Z}_{\alpha}, \tilde{x}_{\alpha}\right)$ since it will be oneto-one from the free generators of $\pi_{n}\left(\widetilde{Z}_{\alpha}, \widetilde{y}_{\alpha}\right)$ into the free generators of $\pi_{n}\left(\widetilde{Z}_{\alpha}, \tilde{x}_{\alpha}\right)$.

ClaIM 9. $\tilde{g}_{\alpha} * \circ p_{\alpha}=0$ for all $\alpha \in \Gamma$.

Proof of Claim 9. According to the proof of Claim $8, \widetilde{g}_{\alpha}$ is an imbedding of $\left(\widetilde{Z}_{\alpha}, \tilde{y}_{\alpha}\right)$ into $\left(\tilde{Z}_{\alpha}, \tilde{x}_{\alpha}\right)$ and $\widetilde{g}_{\alpha}$ takes the free generators of $\pi_{n}\left(\tilde{Z}_{\alpha}, \tilde{y}_{\alpha}\right)$ corresponding to the attached $S^{n}$ 's into the free generators of $\pi_{n}\left(\widetilde{Z}_{\alpha}, \tilde{x}_{\alpha}\right)$ corre- 
sponding to the attached $S^{n}$ 's in $\left(\tilde{Z}_{\alpha}, \tilde{x}_{\alpha}\right)$. Since $\left.\tilde{g}_{\alpha} \tilde{y}_{\alpha}\right)=\tilde{x}_{\alpha}$ and no $S^{n}$ is attached at $\tilde{y}_{\alpha}$, no $S^{n}$ in $\left(\tilde{Z}_{\alpha}, \tilde{y}_{\alpha}\right)$ maps onto the $S^{n}$ attached at $\tilde{x}_{\alpha}=0 \in R^{n} \subset$ $\widetilde{Z}_{\alpha}$ in $\left(\tilde{Z}_{\alpha}, \widetilde{x}_{\alpha}\right)$. By the proof of Claim 7 this implies that $\widetilde{g}_{\alpha^{*}}\left(\pi_{n}\left(\tilde{Z}_{\alpha}, \tilde{y}_{\alpha}\right)\right) \cap$ $\operatorname{im} h_{\alpha}=\{0\}$ in $\pi_{n}\left(\tilde{Z}_{\alpha}, \tilde{x}_{\alpha}\right)$. Now $\tilde{g}_{\alpha} \circ \circ p_{\alpha}=h_{\alpha}$ and thus $\tilde{g}_{\alpha} * p_{\alpha}=0$ as asserted.

The proof of Theorem 4.3 is now complete.

5. Exactness of Čech homology. In this section we use Theorem 2.7 to give an example of a nonmetric movable compact Hausdorff pair $(X, A)$ such that the Cech homology sequence of the pair is not exact. This answers a question posed in [28]. The example shows that the movability of the pair $(X, A)$ in Theorem 1.4 cannot be eliminated if one uses the Mardesic and Segal definition of movability in Definition 1.1.

5.1. ExAmple. Let $A=\operatorname{char} \prod_{i=1}^{\infty} Z$. Then $A$ is a compact connected abelian topological group such that $H^{1}(A) \simeq \mathrm{II}_{i=1}^{\infty} Z$. Now $\Pi_{i=1}^{\infty} Z$ is $\aleph_{1}$-free and thus by Theorem 2.2, $A$ is movable. By Theorem 2.7, $\operatorname{Hom}\left(\Pi_{t=1}^{\infty} Z, Z\right) \simeq$ $\bigoplus_{i=1}^{\infty} Z$ and thus by Theorem $2.4, H_{1}(A) \simeq \bigoplus_{i=1}^{\infty} Z$. Now let $f: A \rightarrow A$ be defined by $f(x)=2 x$ and let $X=M_{f}$ be the mapping cylinder of $f$. Let the subset $B$ of $X$ be the top of the mapping cylinder

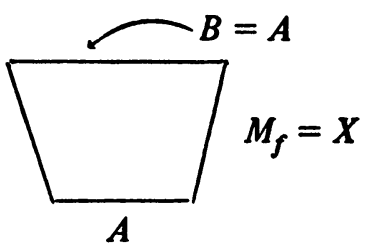

Then $B$ is homeomorphic to $A$ and $X$ is homotopy equivalent to $A$. We claim that $(X, B)$ is a movable pair and that the Cech homology sequence of $(X, B)$ is not exact. Let $\left\{T_{\alpha}^{n} ; \pi_{\alpha \beta} ; \alpha \leqslant \beta \in \Gamma\right\}$ be an inverse system of tori with onto continuous homomorphisms as bonding maps having $A$ as inverse limit. Let $f_{\alpha}: T_{\alpha}^{n_{\alpha}} \rightarrow T_{\alpha}^{n_{\alpha}}$ be defined by $f_{\alpha}(x)=2 x$ and let $X_{\alpha}$ be the mapping cylinder of $f_{\alpha}$. Let $B_{\alpha}$ be the top of the mapping cylinder so that $B_{\alpha}$ is homeomorphic to $T_{\alpha}^{n_{\alpha}}$ and $X_{\alpha}$ is homotopy equivalent to $T_{\alpha}^{n_{\alpha}}$. Now for all $\beta \geqslant \alpha$ the following diagram commutes.

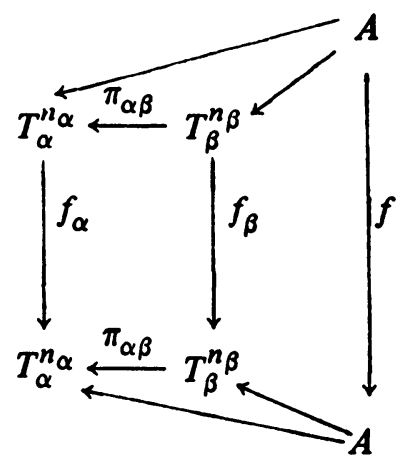


Define $g_{\alpha \beta}: M_{\beta} \rightarrow M_{\alpha}$ so that $g_{\alpha \beta}$ is equal to $f_{\alpha \beta}$ on the top and bottom of the mapping cylinder and at every intermediate level. Then $g_{\alpha \beta}\left(B_{\beta}\right)=B_{\alpha}$ and by the above commutative diagram $g_{\alpha \beta}$ is continuous and $(X, B)$ is the inverse limit of the inverse system $\left\{\left(X_{\alpha}, B_{\alpha}\right) ; g_{\alpha \beta} ; \alpha<\beta \in \Gamma\right\}$.

Claim 1. The system $\left\{\left(X_{\alpha}, B_{\alpha}\right) ; g_{\alpha \beta} ; \alpha \leqslant \beta \in \Gamma\right\}$ is a movable inverse system.

Proof of Claim 1. Let $\alpha \in \Gamma$ and let $\beta \geqslant \alpha$ be the index given by the movability of $A$. Then let $\gamma \geqslant \beta$ be arbitrary and $\gamma^{\beta \gamma}: T_{\beta}^{n \beta} \rightarrow T_{\gamma}^{n} \gamma$ be the map given by the movability of the system $\left\{T_{\alpha}^{n} \alpha\right.$. We may take $r^{\beta \gamma}$ to be a continuous homomorphism and $\pi_{\alpha \beta}=\pi_{\alpha \gamma} \circ r^{\beta \gamma}$. In that case, for every $\gamma \geqslant \beta$ we have the following commutative diagram.

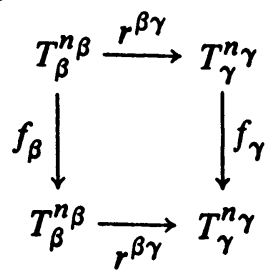

If we define $s^{\beta \gamma}: X_{\beta} \rightarrow X_{\gamma}$ by letting $s^{\beta \gamma}=r^{\beta \gamma}$ at every level of the mapping cylinder, then the above commutative diagram implies that $s^{\beta \gamma}$ is continuous. Since $s^{\beta \gamma}\left(B_{\beta}\right) \subset B_{\gamma}, s^{\beta \gamma}:\left(X_{\beta}, B_{\beta}\right) \rightarrow\left(X_{\gamma}, B_{\gamma}\right)$. Clearly for $\gamma \geqslant \beta, g_{\alpha \beta}=$ $g_{\alpha \gamma} \circ s^{\beta \gamma}$, and $\left\{\left(X_{\alpha}, B_{\alpha}\right)\right\}$ is a movable system.

Now consider the Čech homology sequence:

$$
\cdots \rightarrow H_{n}(X) \rightarrow H_{n}(X, B) \rightarrow H_{n-1}(B) \rightarrow \cdots
$$

We will show that

$$
H_{1}(X) \rightarrow H_{1}(X, B) \rightarrow H_{0}(B)
$$

is not exact. Note that $H_{1}(X) \simeq H_{1}(A)$ and thus $H_{1}(X) \simeq \bigoplus_{i=1}^{\infty} Z$. Also $H_{0}(B)$ $\simeq Z$. Thus $H_{1}(X)$ and $H_{0}(B)$ are both countable groups. If the above sequence were exact, then $H_{1}(X, B)$ would also be countable. However, we claim that $H_{1}(X, B) \simeq \bigoplus_{\lambda \in \Lambda} Z_{2}$ where card $\Lambda=2^{c}$ where $c$ is the cardinality of the real numbers. Thus the sequence cannot be exact. We will show $H_{1}(X, B) \simeq$ $\bigoplus_{\lambda \in \Lambda} Z_{2}$ in the next two claims and the example will be complete.

ClaIM 2. $H_{1}(X, B) \simeq \operatorname{Hom}\left(\Pi_{i=1}^{\infty} Z, Z_{2}\right)$.

Proof of Claim 2. Let $e_{\alpha}^{1}: T_{\alpha}^{n_{\alpha}} \rightarrow B_{\alpha}$ be the natural homeomorphism and $e_{\alpha}^{2}: T_{\alpha}^{n_{\alpha}} \rightarrow M_{f_{\alpha}}=X_{\alpha}$ be the imbedding onto the bottom of the mapping cylinder

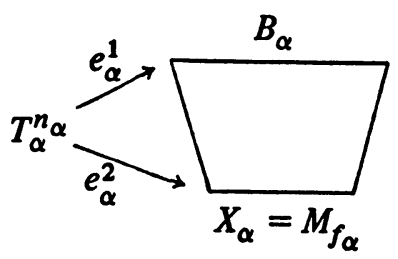


Then the diagram commutes up to homotopy:

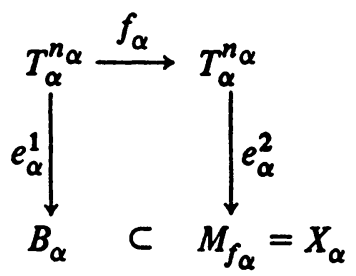

Now $f_{\alpha}^{*}: \operatorname{char} T_{\alpha}^{n_{\alpha}} \rightarrow \operatorname{char} T_{\alpha}^{n_{\alpha}}$ is defined by $2 x$. Thus $f_{\alpha}^{*}: H^{1}\left(T_{\alpha}^{n_{\alpha}}\right) \rightarrow H^{1}\left(T_{\alpha}^{n_{\alpha}}\right)$ is also defined by $2 x$ since char $T_{\alpha}^{n_{\alpha}}$ is naturally isomorphic to $H^{1}\left(T_{\alpha}^{n_{\alpha}}\right)$. By Theorem $2.4, H_{1}\left(T_{\alpha}^{n_{\alpha}}\right)$ is naturally isomorphic to $\operatorname{Hom}\left(H^{1}\left(T_{\alpha}^{n_{\alpha}}\right), Z\right)$ and thus $f_{\alpha^{*}}: H_{1}\left(T_{\alpha}^{n_{\alpha}}\right) \rightarrow H_{1}\left(T_{\alpha}^{n_{\alpha}}\right)$ is also defined by $2 x$. Thus we have the exact sequence:

$$
0 \rightarrow H_{1}\left(B_{\alpha}\right) \stackrel{2 x}{\longrightarrow} H_{1}\left(X_{\alpha}\right) \rightarrow H_{1}\left(X_{\alpha}, B_{\alpha}\right) \stackrel{0}{\rightarrow} H_{0}\left(B_{\alpha}\right) \stackrel{\cong}{\rightrightarrows} H_{0}\left(X_{\alpha}\right) .
$$

This sequence gives us the diagram:

$$
\begin{aligned}
& 0 \rightarrow H_{1}\left(T_{\alpha}^{n_{\alpha}}\right) \stackrel{f_{\alpha}^{*}}{\longrightarrow} H_{1}\left(T_{\alpha}^{n_{\alpha}}\right) \rightarrow H_{1}\left(T_{\alpha}^{n_{\alpha}}\right) / 2 H_{1}\left(T_{\alpha}^{n_{\alpha}}\right) \rightarrow 0 \\
& e_{\alpha^{*}}^{1}=\simeq \quad e_{\alpha^{*}}^{2}=\simeq \downarrow \\
& 0 \longrightarrow H_{1}\left(B_{\alpha}\right) \longrightarrow H_{1}\left(X_{\alpha}\right) \longrightarrow H_{1}\left(X_{\alpha}, B_{\alpha}\right) \longrightarrow 0
\end{aligned}
$$

which is natural. Now let $\tau: \operatorname{Hom}(H, Z) \rightarrow \operatorname{Hom}\left(H, Z_{2}\right)$ be defined for any abelian group $H$ by $\tau(h)=g \circ h$ where $g: Z \rightarrow Z_{2}$ is the modulo 2 homomor. phism. Then for $H$ a torsion free finitely generated abelian group (sic), there is an exact sequence:

$$
0 \rightarrow \operatorname{Hom}(H, Z) \stackrel{2 x}{\longrightarrow} \operatorname{Hom}(H, Z) \stackrel{\tau}{\rightarrow} \operatorname{Hom}\left(H, Z_{2}\right) \rightarrow 0
$$

which is natural in $H$. Combining the last two diagrams together with Theorem 2.4 we have the following diagram:

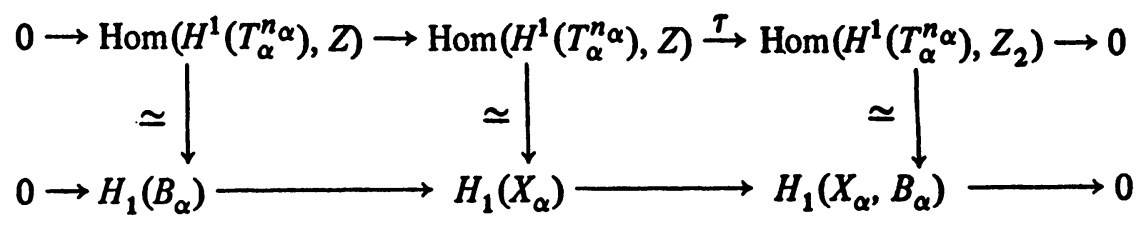

The first two isomorphisms are natural and the exact sequences are natural. Thus the last isomorphism is also natural. This implies that $\lim \left\{\operatorname{Hom}\left(H^{1}\left(T_{\alpha}^{n_{\alpha}}\right), Z_{2}\right)\right\}$ is isomorphic to $\lim _{\leftarrow}\left\{H_{1}\left(X_{\alpha}, B_{\alpha}\right)\right\}=H_{1}(X, B)$. However, $\underset{\leftarrow}{\lim }\left\{\operatorname{Hom}\left(H^{1}\left(T_{\alpha}^{n_{\alpha}}\right), Z_{2}\right)\right\}$ is isomorphic to $\left.\stackrel{\operatorname{Hom}}{\left(\lim _{\longrightarrow}\right.}\left\{H^{1}\left(T_{\alpha}^{n_{\alpha}}\right)\right\}, Z_{2}\right)$. Thus

$$
\operatorname{Hom}\left(H^{1}(A), Z_{2}\right) \simeq H_{1}(X, B) \text {. }
$$

Since $H^{1}(A) \simeq \Pi_{i=1}^{\infty} Z, H_{1}(X, B) \simeq \operatorname{Hom}\left(\Pi_{i=1}^{\infty} Z, Z_{2}\right)$ as asserted. 
Claim 3. $H_{1}(X, B) \simeq \bigoplus_{\lambda \in \Lambda} Z_{2}$ where card $\Lambda=2^{c}$.

Proof of Claim 3. By Claim 2, $H_{1}(X, B) \simeq \operatorname{Hom}\left(\Pi_{i=1}^{\infty} Z, Z_{2}\right)$. Let $h: \Pi_{i=1}^{\infty} Z \rightarrow \Pi_{i=1}^{\infty} Z_{2}$ be defined by $h\left(x_{i}\right)=\left(x_{i} \bmod 2\right)$. Then for any homomorphism $g: \prod_{i=1}^{\infty} Z \rightarrow Z_{2}$ there is a unique homomorphism $\bar{g}: \prod_{i=1}^{\infty} Z_{2} \rightarrow Z_{2}$ such that the diagram commutes.

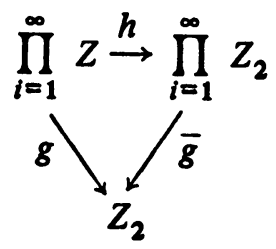

The correspondence $g \rightarrow \bar{g}$ is an isomorphism from $\operatorname{Hom}\left(\Pi_{i=1}^{\infty} Z, Z_{2}\right)$ onto $\operatorname{Hom}\left(\Pi_{i=1}^{\infty} Z_{2}, Z_{2}\right)$. Now $\Pi_{i=1}^{\infty} Z_{2}$ is a vector space over $Z_{2}$ with Hamel dimension $c$. Thus $\Pi_{i=1}^{\infty} Z_{2}$ is isomorphic to $\bigoplus_{\delta \in \Delta} Z_{2}$ where card $\Delta=c$. Thus $\operatorname{Hom}\left(\Pi_{i=1}^{\infty} Z_{2}, Z_{2}\right) \simeq \operatorname{Hom}\left(\bigoplus_{\delta \in \Delta} Z_{2}, Z_{2}\right)$ is isomorphic to $\bigoplus_{\lambda \in \Lambda} Z_{2}$ where card $\Lambda=2^{c}$. Thus Hom $\left(\Pi_{i=1}^{\infty} Z, Z_{2}\right)$ is also isomorphic to $\bigoplus_{\lambda \in \Lambda} Z_{2}$ as asserted.

This completes the proof that the example has the desired properties.

\section{REFERENCES}

1. K. Borsuk, Concerning homotopy properties of compacta, Fund. Math. 62 (1968), 223-254. MR 37 \#4811. \#4925.

2. - On movable compacta, Fund. Math. 66 (1969/70), 137-146. MR 40

3. J. Draper and J. Keesling, An example concerning the Whitehead theorem in shape theory, Fund. Math. (to appear).

4. L. Fuchs, Infinite abelian groups. Vol. I, Pure and Appl. Math., vol. 36, Academic Press, New York and London, 1970. MR 41 \#333. 1973.

5. Infinite abelian groups. Vol. II, Academic Press, New York and London,

6. K. H. Hofmann, Categories with convergence, exponential functors, and the cohomology of compact abelian groups, Math. Z. 104 (1968), 106-140. MR 37 \#4195.

7. W. Holsztynski, An extension and axiomatic characterization of Borsuk's theory of shape, Fund. Math. 70 (1971), 157-168. MR 43 \#8080.

8. J. Keesling, Shape theory and compact connected abelian topological groups, Trans. Amer. Math. Soc. 194 (1974), 349-358.

9. - Continuous mappings induced by shape morphisms, Proc. Amer. Math. Soc. 41 (1973), 315-320.

10. - An algebraic property of the Cech cohomology groups which prevents local connectivity and movability. Trans. Amer. Math. Soc. 190 (1974), 151-162.

11. - On the shape of torus-like continua and compact connected topological groups, Proc. Amer. Math. Soc. 40 (1973), 297-302. MR 47 \#7686.

12. - Products in the shape category and some applications, Convegno di Topologia, Symposia Mathematica (to appear).

13. - On the Whitehead theorem in shape theory, Fund. Math. (to appear).

14. The Cech homology of compact connected abelian topological groups with applications to shape theory, Lecture Notes in Math., vol. 438, Springer-Verlag, New York, 1975, pp. 325-331.

15. The Cech cohomology of movable and n-movable spaces, Trans. Amer. Math. Soc. 219 (1976), 149-167. 
16. G. Kozlowski and J. Segal, n-movable compacta and ANR-systems, Fund. Math. (to appear).

17. Locally well-behaved paracompacta in shape theory (preprint).

18. - Movability and shape connectivity (preprint).

19. K. Kuperberg, An isomorphism of Hurewicz-type in Borsuk's theory of shape, Fund. Math. 77 (1972), 21-32. MR 48 \#3042.

20. S. Mac Lane and G. Birkhoff, Algebra, Macmillan, New York, 1967. MR 35 \#5266.

21. S. Mardešic, Shapes for topological spaces, General Topology and Appl. 3 (1973), 265-282. MR 48 \#2988.

22. - On the Whitehead theorem in shape theory. I (preprint).

23. - On the Whitehead theorem in shape theory. II (preprint).

24. S. Mardešić and J. Segal, Movable compacta and ANR-systems, Bull. Acad. Polon. Sci. Sér. Sci. Math. Astronom. Phys. 18 (1970), 649-654. MR 44 \#1026.

25. - Shapes of compacta and ANR-systems, Fund. Math. 72 (1971), 41-59. MR 45 \#7686.

26. M. Moszyńska, Uniformly movable compact spaces and their algebraic properties, Fund. Math. 77 (1972), 125-144. MR 48 \#1224.

27. The Whitehead theorem in the theory of shapes, Fund. Math. 80 (1973), 221-263. MR 49 \#3922.

28. R. H. Overton, Cech homology for movable compacta, Fund. Math. 77 (1973), 241-251.

29. R. H. Overton and J. Segal, A new construction of movable compacta, Glasnik Mat. Ser. III 6 (26) (1971), 361-363. MR 48 \#1157.

30. L. S. Pontrjagin, Continuous groups, 2nd ed., GITTL, Moscow, 1954; English transl., Topological groups, Gordon and Breach, New York, 1966. MR 34 \#1439. \#1007.

31. E. H. Spanier, Algebraic topology, McGraw-Hill, New York, 1966. MR 35

32. S. Spiez, Movability and uniform movability, Bull. Acad. Polon. Sci. Sér. Sci. Math. Astronom. Phys. 18 (1970), 649-654.

DEPARTMENT OF MATHEMATICS, UNIVERSITY OF FLORIDA, GAINESVILLE, FLORIDA 32611 